\title{
Spectra and eigenvectors of scale-free networks
}

\author{
K.-I. Goh, B. Kahng, and D. Kim \\ Center for Theoretical Physics and School of Physics, Seoul National University, Seoul 151-742, Korea
}

(May 28, 2018)

\begin{abstract}
We study the spectra and eigenvectors of the adjacency matrices of scale-free networks when bi-directional interaction is allowed, so that the adjacency matrix is real and symmetric. The spectral density shows an exponential decay around the center, followed by power-law long tails at both spectrum edges. The largest eigenvalue $\lambda_{1}$ depends on system size $N$ as $\lambda_{1} \sim N^{1 / 4}$ for large $N$, and the corresponding eigenfunction is strongly localized at the hub, the vertex with largest degree. The component of the normalized eigenfunction at the hub is of order unity. We also find that the mass gap scales as $N^{-0.68}$.

PACS numbers: 05.10.-a, 05-40.a, 05-50.+q, 87.18.Sn
\end{abstract}

Complex systems such as social, biological, and economic systems consist of many constituents such as individuals, substrates, and companies, respectively [1]. Each constituent reacts and adapts to the pattern created in the system through diverse interactions. Cooperative phenomena between constituents in such complex systems may be described in terms of random graphs, consisting of vertices and edges, where vertices (edges) represent constituents (their interactions). The study of complex systems in terms of random graph was initiated by Erdös and Rényi (ER) [2]. In the ER model, the number of vertices is fixed, and edges connecting from one vertex to another occur with probability $p$. Then there exists a probability threshold $p_{c}$, above which the system is percolated. Recently Watts and Strogatz (WS) introduced the small-world network [3] to consider local clustering, while the number of vertices is also fixed. The WS model offers the first indication that real networks could be more complex than predicted by the ER model.

Recently Barabási and Albert (BA) 化 introduced an evolving model where the number of vertices increases linearly with time rather than fixed, and a newly introduced vertex is connected to $m$ already existing vertices, following the so-called preferential attachment rule that the vertices with more edges are preferentially selected for the connection to the new vertex. The number of edges $k$ incident upon a vertex is the degree of the vertex. Then the degree distribution $P(k)$ of vertices, equivalent to the connectivity distribution, follows a power-law $P(k) \sim k^{-3}$ for the BA model, while for the ER and WS models, it follows a Poisson distribution. The BA model is interesting in a sense that a lot of complex interactive networks such as the world wide web $[.5,6]$, the actor network 沟, the citation network of scientific papers and the author collaboration network [7], metabolic network [8] and food web [9] in biological systems all exhibit the power-law in the degree distribution, implying that a characteristic degree is absent in such systems. Thus the BA model is called a scale-free network. In the BA network, one may assign a direction on each edge pointing from the younger vertex to the older one 10]. However, when the direction on each edge is ignored, allowing bi-directional interactions such as download and upload communications in Internet, the BA network may be regarded as a simple model for the Internet topology [11].

While it is known that the BA network follows the power-law in its degree distribution, further structural properties are not so well known. When $m=1$, the BA network forms a tree structure without forming any loop, but for $m>1$, loops are formed, and network topology becomes much complicated. So it would be interesting to investigate the spectrum of the BA network, because generally the spectrum of a random graph and corresponding eigenvectors are closely related to topological features of the random graph [13 15]. In this paper, we study the spectrum and the corresponding eigenvectors of the adjacency matrix of the BA network, comparing spectral properties with structural features. When a BA network is composed of $N$ vertices, the adjacency matrix A consists of $N \times N$ elements, $\left\{a_{i, j}\right\}(i, j=1, \ldots, N)$, defined as $a_{i, j}=1$ if vertices $i$ and $j$ are connected via an edge, and $a_{i, j}=0$ otherwise. $k_{i}=\sum_{j} a_{i, j}$ is the degree of the vertex $i$. In the BA model, the vertex with the largest degree is singled out and is called the hub, denoted by $h$ hereafter, in this work. From previous studies [4], we know that $k_{h}$, the degree of the hub, scales as $k_{h} \sim m N^{1 / 2}$. The vertices are ordered in their ages; the vertex $i$ is the one born at time $i$.

Since we consider the bi-directional case (sometimes called the undirected case), the adjacency matrix $\mathbf{A}$ is real and symmetric, so that all eigenvalues are real and the largest eigenvalue is not degenerate. We obtained the spectrum of the BA network via exact diagonalization for $N$ up to 5,000 and for the first few largest eigenvalues, via the Lanczos method [12] for $N$ up to 400,000. Throughout this paper, numerical simulations were carried out for $m=2$, the simplest case including the loop structure.

Eigenvalues- We consider the distribution of the 
eigenvalues, shown in Fig.1. Analytic formula for the spectrum is not known yet, but one can easily see that the spectrum does not fit to the semi-circular equation derived by Wigner [13] appropriate to the ER random graph. The density of eigenvalues $\rho(\lambda)$ in the middle part of the spectrum is likely to fit to the formula, $\rho(\lambda) \sim \exp (-|\lambda| / a)$ where $a \approx 1.25$ (see the left inset of Fig.1), while the density further out follows the powerlaw, $\rho(\lambda) \sim|\lambda|^{-4}$, (see the right inset of Fig.1). Since the power-law decays much slower than the exponential one, the spectrum shows long tails at both spectrum edges (see Fig.1). Such behavior has also been observed in the financial time series [16].

The size dependence of the largest eigenvalue $\lambda_{1}$ is of interest and we found numerically that $\lambda_{1}$ increases as $\sim N^{1 / 4}$ for large systems (see Fig.2). To consider the relation between the largest eigenvalue $\lambda_{1}$ and the structure of the BA network, we consider the followings. Let $\mathbf{x}$ and $\mathbf{y}$ be $N \times 1$ column vectors, related through

$$
\mathbf{y}(n)=\mathbf{A}^{n} \mathbf{x}
$$

Let $v_{i, l}$ be the $i$-th component of the normalized eigenvector corresponding to the $l$-th eigenvalue of $\mathbf{A}, \lambda_{l}$, $\left(\lambda_{1}>\lambda_{2} \geq \ldots \geq \lambda_{N}\right)$. Then Eq.(1) may be rewritten as

$$
y_{i}(n)=\sum_{l} \sum_{j} \lambda_{l}^{n} v_{i, l} v_{j, l} x_{j}
$$

When we set $x_{j}=1$ for some $j$ and $x_{r}=0$ for $r \neq j, y_{i}(n)$ becomes the number of possible ways of $n$-step walks starting from the vertex $j$ and terminating at the vertex $i$. This will be denoted as $y_{j \rightarrow i}(n)$ hereafter. In particular, when $i=j, y_{j \rightarrow j}(n)$ is the number of possible ways to return to the starting vertex $j$ after $n$-step walks. When $n$ is sufficiently large, $y_{j \rightarrow i}(n)$ can be represented in terms of the largest eigenvalue $\lambda_{1}$ alone as

$$
y_{j \rightarrow i}(n) \approx \lambda_{1}^{n} v_{i, 1} v_{j, 1}
$$

In particular, when $i=j$,

$$
y_{j \rightarrow j}(n) \approx \lambda_{1}^{n} v_{j, 1}^{2}
$$

Even though the relation Eq.(4) holds for any vertex $j$, we consider it particularly at the hub. Now let us consider the number of ways of $n+2$ steps returning to the starting vertex $h$. It can be split into two parts,

$$
y_{h \rightarrow h}(n+2)=y_{h \rightarrow h}(n) y_{h \rightarrow h}(2)+\sum_{j \neq h} y_{h \rightarrow j}(n) y_{j \rightarrow h}(2) \text {. }
$$

According to Eq.(4), $\lambda_{1}^{2} \approx \frac{y_{h \rightarrow h}(n+2)}{y_{h \rightarrow h}(n)}=y_{h \rightarrow h}(2)+\frac{\sum_{j \neq h} y_{h \rightarrow j}(n) y_{j \rightarrow h}(2)}{y_{h \rightarrow h}(n)}$,

where $y_{h \rightarrow h}(2)$ corresponds to the degree of the hub, $y_{h \rightarrow h}(2)=m \sqrt{N}$, while $y_{j \rightarrow h}(2)$ is found numerically to behave as $\sim N^{0.05} / j^{0.43}$, weakly depending on $N$ (see Fig.3). The second term of the right hand side of Eq.(6) can be written using Eqs.(3) and (4) as $\sum_{j \neq h}\left(\frac{v_{j, 1}}{v_{h, 1}}\right) y_{j \rightarrow h}(2)$. We will show later that $v_{h, 1} \approx 1 / 2$, while $v_{j, 1} \sim(N j)^{-1 / 4}$ for $j$ being away from $h$, leading to the result that the second term becomes $\mathcal{O}\left(N^{0.1}\right)$. Consequently, $\lambda_{1}$ is contributed dominantly by the first term in the right hand side of Eq.(6), leading to $\sim m^{1 / 2} N^{1 / 4}$.

The result of $\lambda_{1} \sim N^{1 / 4}$ can also be understood through two toy models. First, one vertex is located at the center, and all the other $N-1$ vertices are linked only to the center vertex (Fig.4a). This structure, called the radial structure, is an extreme case of "winner-takes-all". The largest eigenvalue of this structure is $\lambda_{1}=\sqrt{N-1}$, while the largest degree is $N-1$. Second, we consider a two-level Cayley tree structure (Fig.4b). When the coordination number is chosen as $k=\sqrt{N}$, the total number of vertices becomes $N+1$, and the largest degree $\sqrt{N}$, as in the case of the BA network. The largest eigenvalue for this toy model is found to be $\lambda_{1}=\sqrt{2 k-1} \sim N^{1 / 4}$. So, in both models, $\lambda_{1} \sim \sqrt{k_{h}}$.

The second largest eigenvalue in its absolute magnitude is located on the negative side of the spectrum. The absolute magnitude of this eigenvalue $\left|\lambda_{N}\right|$ is also found to scale as $\left|\lambda_{N}\right| \sim N^{1 / 4}$ (see Fig.2). The difference between the first two largest eigenvalues in their absolute magnitude, $\lambda_{1}-\left|\lambda_{N}\right|$, is found to scale as $\sim N^{-0.43}$ (see the inset of Fig.2). Thus, the mass gap, defined as $\log \left(\lambda_{1} /\left|\lambda_{N}\right|\right)$ [17], scales as $\sim N^{-z}$ with $z \approx 0.68$.

Next, we consider the size dependence of the moments of the spectrum. First, since the matrix A is traceless, the first moment becomes zero, that is, $\mathcal{M}_{1}=\sum_{i}^{N} \lambda_{i}=0$. Second, for each vertex, the number of ways to return to the starting vertex by a 2-step walk is the same as the degree of that vertex, so that $\mathcal{M}_{2}=\sum_{i}^{N} \lambda_{i}^{2}=2 m N$. Third, since the path to return to the starting vertex by a 3 -step walk forms a triangle, the total number of triangles $\mathcal{T}_{N}$ in the system can be obtained through the third moment, $\mathcal{M}_{3}=\sum_{i}^{N} \lambda_{i}^{3}=6 \mathcal{T}_{N}$. We found numerically that $\mathcal{M}_{3} \sim N^{0.40}$ (see Fig.5). Note that while the first moment vanishes, the third moment does not, implying that the spectrum is not completely symmetric with respect to $\lambda=0$.

Eigenvector- Since the adjacency matrix $\mathbf{A}$ is real and symmetric, every component of the eigenvector corresponding to the largest eigenvalue is positive. Since the 
largest eigenvalue and the corresponding eigenfunction is important, as in the case of Eq.(3), we focus on the eigenvector $\left\{v_{j, 1}\right\}$ for the largest eigenvalue. We consider the square of each component of the eigenvector $\left\{v_{j, 1}^{2}\right\}$ instead of $\left\{v_{j, 1}\right\}$ itself because of the normalization, $\sum_{j} v_{j, 1}^{2}=1$, and compare it with the normalized degree of each vertex $\left\{k_{j} / \sum_{l} k_{l}\right\}$. As seen in Fig.6, the two quantities are correlated in such a way that up-down behavior occurs in a similar fashion.

The components $\left\{v_{j, 1}^{2}\right\}(j=1, \ldots, N)$ is strongly localized at the hub. This is in contrast to the ER case where the corresponding eigenfunction is extended over all vertices (see the inset of Fig.6). We found that $v_{h, 1}^{2}$ at the hub increases with increasing $N$ for small $N$, but converges to a constant close to $1 / 2$ for large $N$ (see Fig.7). The value $1 / 2$ can be obtained from the radial structure analytically. Since the radial structure is the extreme case that the connectivity is mostly localized at the hub, the value $1 / 2$ can become the upper bound of $v_{h, 1}^{2}$. The result that $v_{h, 1}^{2}$ approaches a constant as $N \rightarrow \infty$ is interesting, because $v_{h, 1}^{2}$ behaves differently from the normalized degree at the hub, decreasing as $\sim N^{-1 / 2}$ (see Fig.7). Since the number of returning $n$ step walks from a vertex $j$ is $y_{j \rightarrow j}(n) \propto v_{j, 1}^{2}$ from Eq.(4), $v_{j, 1}^{2}$ can be considered as a measure of contribution from the vertex $j$ to transport processes in a network. The fact that $v_{h, 1}^{2} \sim \mathcal{O}(1)$ can be interpreted as that the hub plays much more dominant role in transports on the BA network, compared with the contribution measured by the normalized degree at the hub, which is $\mathcal{O}(1 / \sqrt{N})$. Thus, the presence of the hub in the scale-free networks diversifies pathways, and enhances the efficiency of transport, while the network systems are vulnerable to the attack on the hub [18]. On the other hand, for other components of the eigenfunction, $\left\{v_{j, 1}^{2}\right\},(j=1, \ldots, N$, but $j \neq h)$, it was found numerically that $v_{j, 1}^{2} \sim 1 / 4 \sqrt{N j}$ (see Fig.8).

Each component of eigenvectors can be obtained through the diagonalization of the adjacency matrix $\mathbf{A}$. However, the eigenfunction for $\lambda_{1}$ can be obtained using the relation, Eq.(4). The knowledge of $y_{j \rightarrow j}(n)$ and $\lambda_{1}$ enables one to obtain each component of the eigenfunction through $v_{j, 1}^{2} \approx y_{j \rightarrow j}(n) / \lambda_{1}^{n}$ for sufficiently large $n$. However, when $n$ is not large enough, $y_{j \rightarrow j}(n)$ for vertex $j$ is affected by local topology around $j$, so that the value of $v_{j, 1}^{2}$ obtained by this method would be different from that by the exact diagonalization. Thus, we can define a characteristic number of steps $n_{c}$, such that for $n>n_{c}$, Eq.(4) holds, while for $n<n_{c}$, it breaks down. To find $n_{c}$, we introduce the quantity,

$$
\delta_{n} \equiv\left|\sum_{j}\left(\frac{y_{j \rightarrow j}(n)}{\lambda_{1}^{n}}-v_{j, 1}^{2}\right)\right|,
$$

which is found to decay exponentially as $\delta_{n} \sim$ $\exp \left(-n / n_{c}\right)$ (see the inset of Fig.9). We found numerically that $n_{c}$ shows a crossover across a characteristic system size $N_{c}$ such that for $N<N_{c}, n_{c} \sim N^{0.35}$, while for $N>N_{c}, n_{c} \sim N^{0.50}$ (see Fig.9). On the other hand, Eq.(7) may be rewritten as $\delta_{n}=\left|\sum_{l>1}\left(\lambda_{l} / \lambda_{1}\right)^{n}\right|$. Then, for sufficiently large $n, \delta_{n}$ is contributed dominantly by the term $\left(\left|\lambda_{N}\right| / \lambda_{1}\right)^{n}$ alone. Combined with $\delta_{n} \sim \exp \left(-n / n_{c}\right)$, one obtains that $n_{c}=\lambda_{1} /\left(\lambda_{1}-\left|\lambda_{N}\right|\right)$, equivalent to the inverse of the mass gap. Thus, $n_{c} \sim N^{z}$. However, the numerical value of the exponent $z$ obtained in this way, $z \approx 0.50$, deviates from the value $z \approx 0.68$ obtained by the direct measurement of $\lambda_{1}-\left|\lambda_{N}\right|$. This can be attributed to the fact that $\delta_{n}$ includes contributions from other eigenvalues.

Shortest paths - Since the role of the hub in transport is much more dominant, compared with the contribution by the normalized degree, we also study topological feature of shortest paths between two vertices [19]. The transport from one position to another is mainly carried along the shortest path(s) between them, and is contributed dominantly by the largest eigenvalue of the adjacency matrix $\mathbf{A}$. We define a set, composed of the vertices on the shortest path(s) from one position to another. Then, since there are $N(N-1) / 2$ pairs of vertices, the same number of sets exist in the system. Among them, we are interested in how many number of different sets a certain vertex $j$ belongs to. This number is called the involving number $P_{j}$, while the normalized involving number is defined as $p_{j} \equiv P_{j} / \sum_{l} P_{l}$ for each vertex $j$. Fig.6 also shows $p_{j}$ versus $j$. It behaves similarly to the up-down behavior of $v_{j, 1}^{2}$ and $k_{j}$. In particular, the involving number at the hub $P_{h}$ is found numerically to scale as $P_{h} \sim N^{2}$, while the total involving number summed over all vertices is found numerically to scale as $\sum_{j=1}^{N} P_{j} \sim N^{2} \log N$ (see Fig.10) [20]. So the normalized involving number at the hub $p_{h} \equiv P_{h} / \sum_{j} P_{j}$ behaves as $p_{h} \sim 1 / \log N$, decaying much more slowly, compared with the normalized degree decreasing as $\sim N^{-0.5}$. This weak dependence on $N$ is comparable to the result that $v_{h, 1} \sim \mathcal{O}(1)$ for large $N$. Therefore, the contribution of the hub to shortest paths is much larger than that of the naive estimate based on the normalized degree at the hub, $\mathcal{O}(1 / \sqrt{N})$.

In conclusion, we have considered the spectrum and eigenvectors of the adjacency matrix of the BA network, when bi-directional interaction is allowed. The density of the eigenvalues decays exponentially for small $|\lambda| \mathrm{s}$, followed by power-law tails at both spectrum edges. This is different from the Wigner's formula appropriate to random graphs. We found that the largest two eigenvalues $\lambda_{1}$ and $\left|\lambda_{N}\right|$ depend on system size $N$ as $\sim N^{1 / 4}$ for large $N$, and the mass gap scales as $N^{-0.68}$. The eigenfunction corresponding to the largest eigenvalue $\lambda_{1}$ is strongly localized at the vertex with the largest degree, called the hub. The component of the normalized eigenfunction for $\lambda_{1}$ at the hub is independent of $N$, implying that the role of the hub in transport on the scale-free 
network becomes much important for larger system, and its contribution becomes much more dominant than expected according to the normalized degree at the hub, which scales as $N^{-1 / 2}$. Therefore, it is very efficient in communication networks to construct central vertices, through which most of information traffic pass.

We would like to thank S.Y. Park for providing us with the Lanczos algorithm codes. This work is supported by grants No.2000-2-11200-002-3 from the BRP program of the KOSEF. While writing this manuscript we learned of a work by Farkas et al. [21], which overlaps some of our results.

[1] N. Goldenfeld and L.P. Kadanoff, Science 284, 87 (1999).

[2] P. Erdös and A. Rényi, Publ. Math. Inst. Hung. Acad. Sci., Ser. A 5, 17 (1960).

[3] D.J. Watts and S.H. Strogatz, Nature 393, 440 (1998); M.E.J. Newman and D.J. Watts, Phys. Lett. A 263, 341 (1999); M.E.J. Newman, S.H. Strogatz, and D.J. Watts, (cond-mat/0007235).

[4] A.-L. Barabási and R. Albert, Science 286, 509 (1999); A.-L. Barabási, R. Albert and H. Jeong, Physica A 272, 173 (1999); ibid 281, 69 (2000).

[5] A. Broder, et al., Proc. of the 9th WWW conference (Amsterdam,2000), 309.

[6] R. Albert, H. Jeong, and A.-L. Barabási, Nature 401, 130 (1999).

[7] S. Redner, Eur. Phys. J. B 4, 131 (1998); M.E.J. Newman, (cond-mat/0007214).

[8] H. Jeong, B. Tombor, R. Albert, Z.N. Oltvani, and A.-L. Barabási, Nature 407, 651 (2000).

[9] R.V. Solé and José Montoya, (cond-mat/0011196).

[10] P.L. Krapivsky, S. Redner, and F. Leyvraz, Phys. Rev. Lett. 85, 4629 (2000); S.N. Dorogovtsev, J.F.F. Mendes, A.N. Samukhin, ibid, 85, 4633 (2000).

[11] E.W. Zegura, K.L. Calvert, and M.J. Donahoo, IEEE/ACM Trans. Network 5, 770 (1997).

[12] C. Lanczos, J. Res. Nat. Bur. Stand 45, 255 (1950); R.B. Lehoucq, D.C. Sorensen, and C. Yang, ARPACK Users' Guide, available at http://www.caam. rice.edu/software/ARPACK.

[13] M. Mehta, Random Matrices (Academic Press, New York, 1995).

[14] B. Bollobás, Random Graphs (Academic Press, New York, 1985).

[15] D.M. Cvetković, M. Doob, and H. Sachs, Spectra of Graphs (Academic Press, New York, 1979).

[16] L. Laloux, P. Cizeau, J.-P. Bouchaud, and M. Potters, Phys. Rev. Lett. 83, 1467 (1999); V. Plerou, P. Gopikrishnan, B. Rosenow, L. A. N. Amaral, H. E. Stanley, ibid, 83, 1471 (1999); J.D. Noh, Phys. Rev. E 61, 5981 (2000).

[17] L.H. Gwa and H. Spohn, Phys. Rev. Lett. 68, 725 (1992); Phys. Rev. A 46, 844 (1992); D. Kim, Phys. Rev. E 52, 3512 (1995).
[18] R. Albert, H. Jeong, and A.-L. Barabási, Nature, 406, 378 (2000); R. Cohen, K. Erez, D. ben-Avraham, and S. Havlin, Phys. Rev. Lett. 85, 4626 (2000).

[19] Z. Alexandrowicz, Phys. Lett. A 80, 284 (1980); J.S. Andrade, et al., Phys. Rev. E 62, 8270 (2000).

[20] The numberical data may also be interpreted to follow a power-law $\sum_{j} P_{j} \sim N^{2.15}$.

[21] I.J. Farkas, I. Derényi, A.-L. Barabási, and T. Vicsek, (cond-mat/0102335).

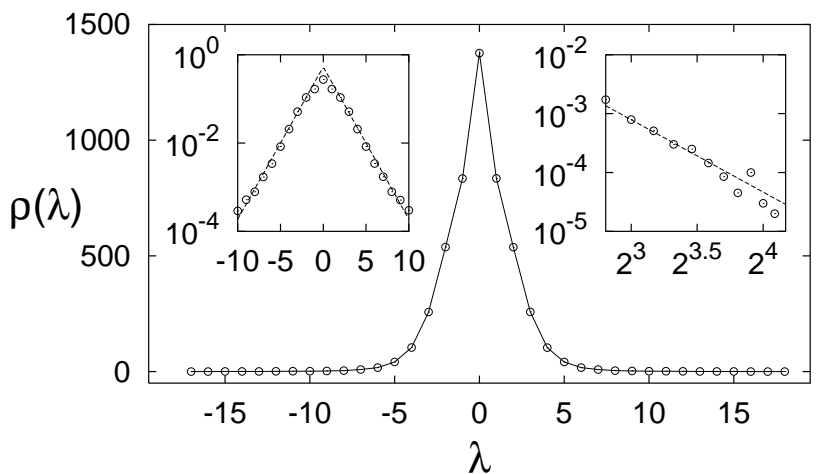

FIG. 1. Plot of the density of eigenvalues of the adjacency matrix A versus eigenvalues for system size $N=5,000$ averaged over 40 configurations. Left inset: Semi-logarithmic plot of the density versus eigenvalues to show the exponential decay for small $|\lambda|$ s. Right inset: Double logarithmic plot of the density versus eigenvalues to show the power-law decay at spectrum edge.

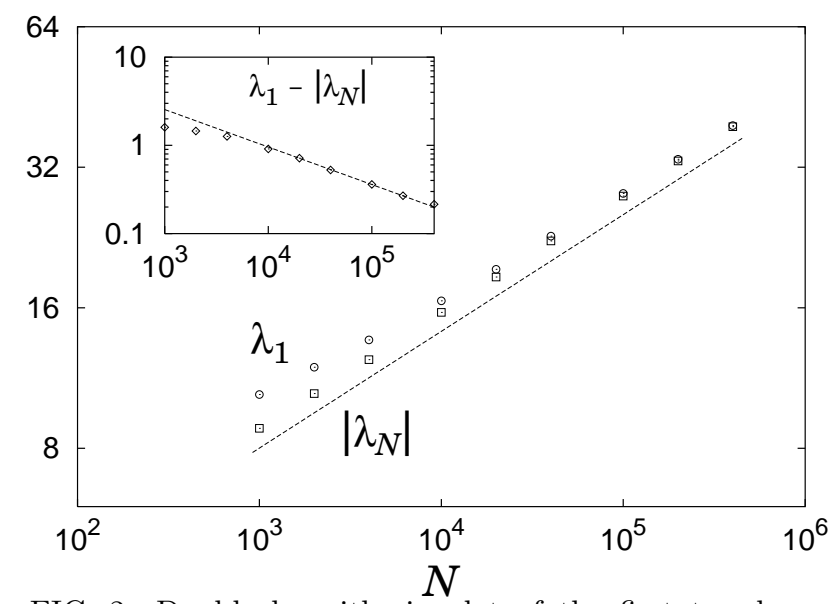

FIG. 2. Double logarithmic plot of the first two largest eigenvalues $\lambda_{1}(\circ)$ and $\left|\lambda_{N}\right|(\square)$ versus system size $N$. The dashed line has slope 0.25 , drawn for the eye. Inset: Double logarithmic plot of the difference, $\lambda_{1}-\left|\lambda_{N}\right|$, versus system size $N$. 


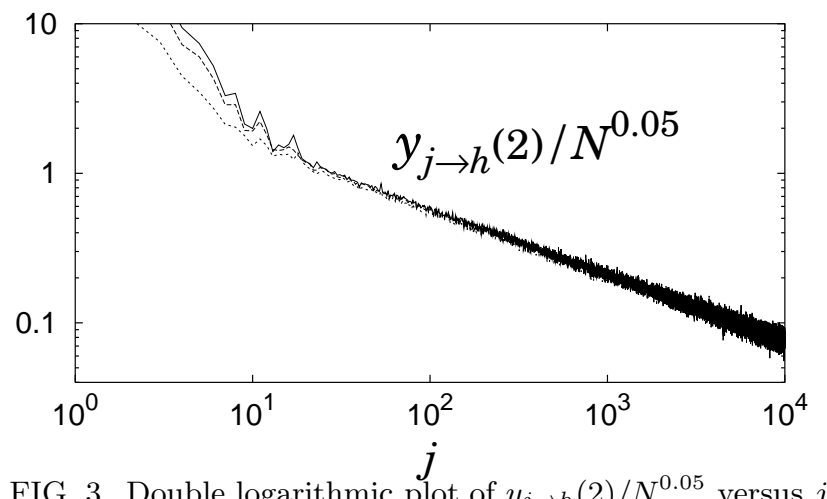

FIG. 3. Double logarithmic plot of $y_{j \rightarrow h}(2) / N^{0.05}$ versus $j$ for different systems $N=1,000,5,000$ and 10,000. The slope at the tail is estimated to be -0.43 .

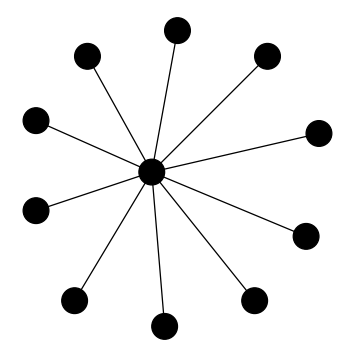

(a)

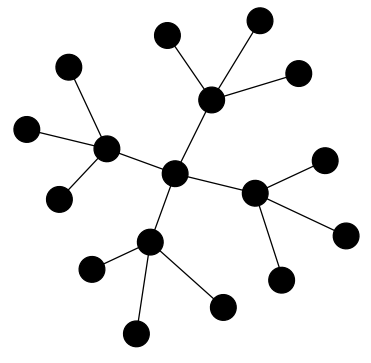

(b)
FIG. 4. The radial structure (a), and the two-level Cayley tree structure with the coordination number $k=4$ (b).

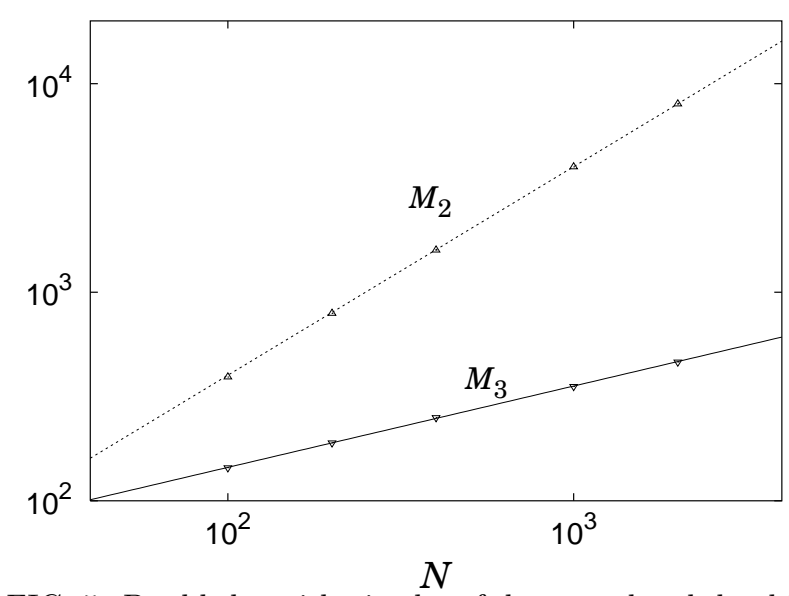

FIG. 5. Double logarithmic plot of the second and the third moments $\mathcal{M}_{2}$ and $\mathcal{M}_{3}$ versus system size $N$. The dotted (solid) line has slope 1.0 (0.40), drawn for the eye.

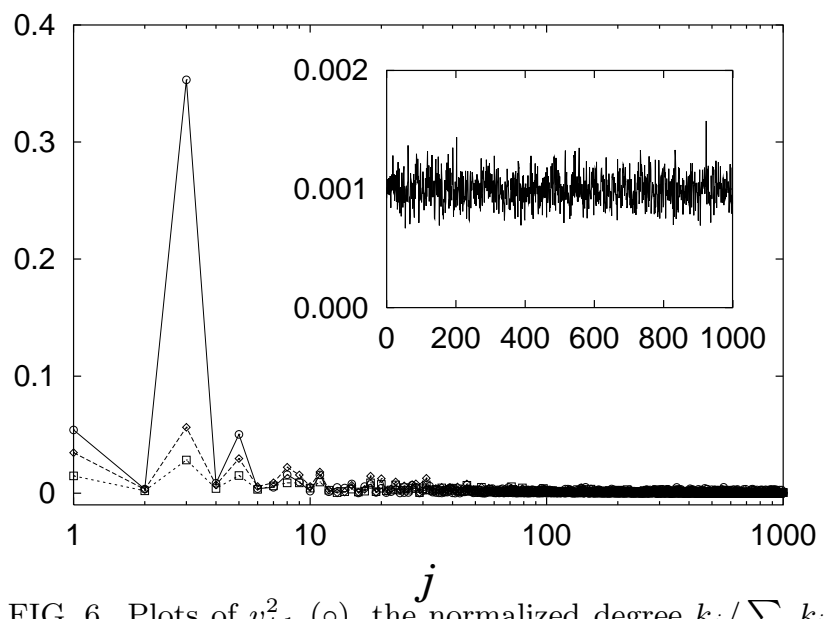

FIG. 6. Plots of $v_{j, 1}^{2}$ (०), the normalized degree $k_{j} / \sum_{i} k_{i}$ $(\square)$, and the normalized involving number $p_{j}(\diamond)$ versus vertex index $j$ for $N=10^{3}$. Inset: The eigenfunction corresponding to the largest eigenvalue for the ER random network for $N=10^{3}$, showing that the eigenfunction is extended.

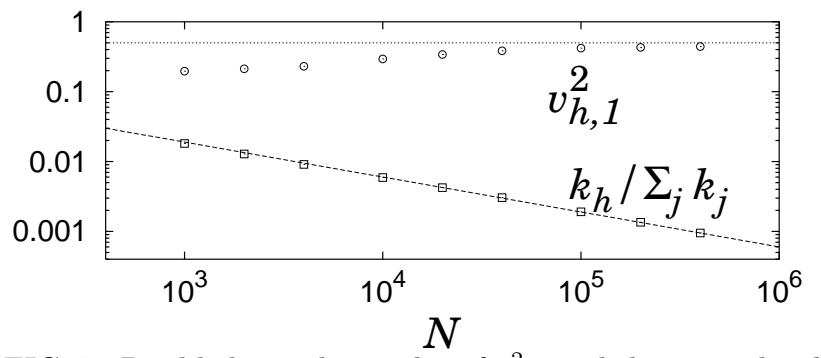

FIG. 7. Double logarithmic plot of $v_{h, 1}^{2}$ and the normalized degree $k_{h} / \sum_{j} k_{j}$ at the hub versus system size $N$. The dotted line 0.5 is an asymptotic line of $v_{h, 1}^{2}$. The dashed line for the normalized degree has slope -0.5 , drawn for the eye.

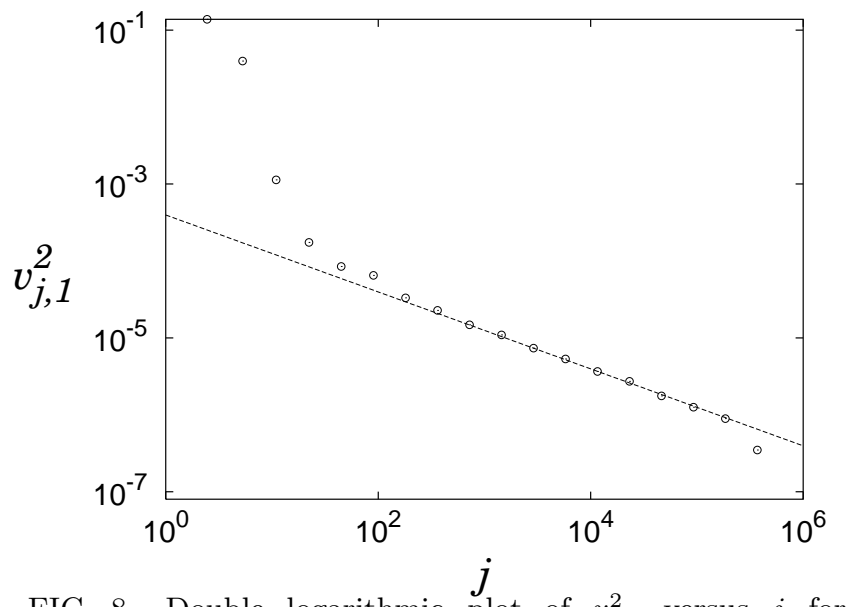

FIG. 8. Double logarithmic plot of $v_{j, 1}^{2}$ versus $j$ for $N=400,000$. The dashed line has slope -0.5 , drawn for the eye. 


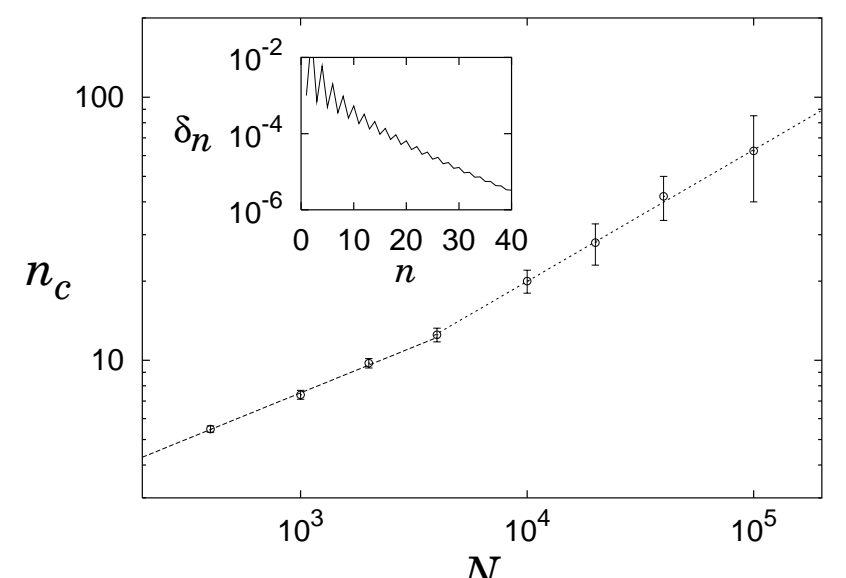

FIG. 9. Double logarithmic plot of $n_{c}$ versus system size $N$. The dashed line has slope 0.35 up to $N_{c} \approx 5,000$, and 0.50 beyond $N_{c}$, drawn for the eye. Inset: Semi-logarithmic plot of $\delta_{n}$ versus number of steps $n$.

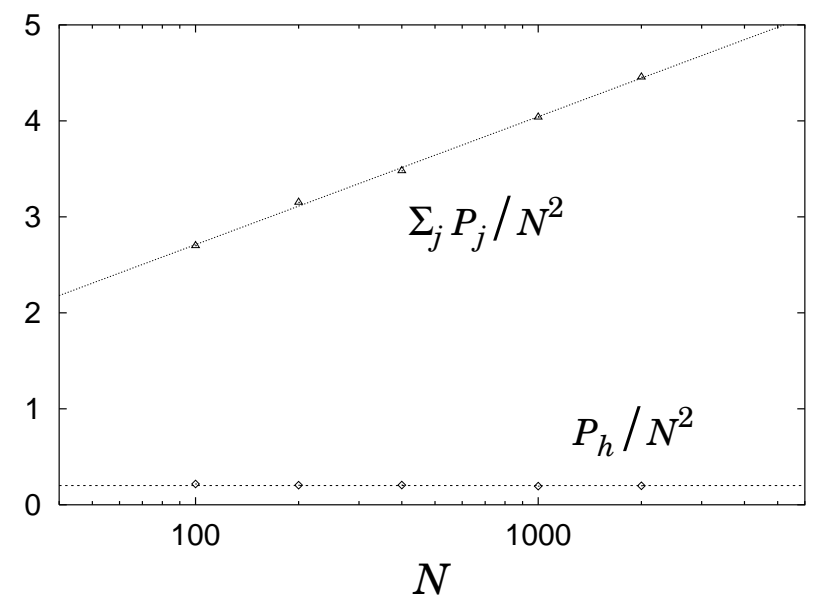

FIG. 10. Semi-logarithmic plot of $P_{h} / N^{2}$ and $\sum_{j} P_{j} / N^{2}$. 